\title{
LEFSCHETZ DECOMPOSITIONS FOR QUOTIENT VARIETIES
}

\author{
REZA AKHTAR AND ROY JOSHUA
}

\begin{abstract}
In an earlier paper, the authors constructed an explicit Chow-Künneth decomposition for the quotient of an abelian varieties by the action of a finite group. In the present paper, the authors extend the techniques used there to obtain an explicit Lefschetz decomposition for such quotient varieties.
\end{abstract}

\section{Introduction}

The notion of a motive, conceived initially by Grothendieck, and later developed further by Manin [15], Kleiman [12] and others, involves replacing the category of smooth projective varieties over a field $k$ with another

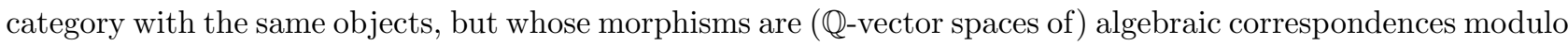
some adequate equivalence relation; that is, some equivalence relation preserved by pullbacks, push-forwards, and intersection with arbitrary cycles. If one chooses rational equivalence - the finest adequate equivalence relation - one obtains the category of Chow motives, and it is natural to ask to what extent one can prove in this category the properties which Grothendieck predicted that motives should enjoy.

In particular, one may ask if a smooth projective variety $X$ (of dimension $d$ ) has a Chow-Künneth decomposition; that is, if the diagonal class $\left[\Delta_{X}\right] \in C H^{d}\left(X \times_{k} X ; \mathbb{Q}\right)$ has a decomposition $\left[\Delta_{X}\right]=\bigoplus_{i=0}^{2 d} \pi_{i}$ into mutually orthogonal idempotents $\pi_{i}$ whose images under the cycle map $C H^{i}\left(X \times_{k} X ; \mathbb{Q}\right) \rightarrow H^{2 i}\left(X \times_{k} X ; \mathbb{Q}\right)$ are the ordinary Künneth components with respect to the Weil cohomology theory $H^{*}$. At present, Chow-Künneth decompositions are known to exist for curves and projective spaces [15], surfaces [17], abelian varieties ([6], [20]), varieties with "finite-dimensional" motives whose Kunneth components of the diagonal are algebraic [11], and several other special classes.

Murre [18] has conjectured that every smooth projective variety $X$ over a field $k$ has a Chow-Künneth decomposition with two further properties: (a) the projectors $\pi_{0}, \ldots, \pi_{j-1}$ and $\pi_{j+d+1}, \ldots, \pi_{2 d}$ should act as zero on the group $C H^{j}(X ; \mathbb{Q})$, and (b) when $2 j+1 \leq i \leq j+d, \pi_{i}$ should act as zero on $C H^{j}(X)$. In [6], Deninger and Murre [6] construct a Chow-Künneth decomposition for abelian varieties; Murre [18] then showed that (a) is satisfied in this case. Moreover, (b) is equivalent to Beauville's conjecture that the eigenspaces $C H_{s}^{i}(A ; \mathbb{Q})$ vanish for $s<0$; see section 2 for explanation of the notation. Since Beauville's conjecture is known to hold for abelian varieties of dimension at most four (cf. [4]), it follows that Murre's conjecture holds for such abelian varieties.

In an earlier paper [1], the authors of this article showed that if $G$ is a finite group acting on an abelian variety $A$, then the Deninger-Murre Chow-Künneth decomposition for $A$ can be used to construct a Chow-Künneth decomposition for the quotient variety $A / G$, and that if (a) and (b) hold for $A$, then they also hold for $A / G$. Thus, Murre's conjecture also holds for such quotients of abelian varieties of dimension at most four. We note that $A / G$ is not in general smooth; however, finiteness of $G$ ensures that the machinery of intersection theory and Chow motives can be extended to varieties of this sort, which we henceforth refer to as pseudo-smooth.

The second author thanks the IHES, the MPI (Bonn) and the NSA for support. 
In [14], Künnemann constructs a Lefschetz decomposition for Chow motives of abelian schemes with respect to the Deninger-Murre Chow-Künneth decomposition. The aim of the present article is to construct an explicit Lefschetz decomposition for the Chow motive of the quotient of an abelian variety by a finite group action with respect to the Chow-Künneth decomposition constructed in our earlier work [1].

Pedrini and Guletskii [7] have shown that if the motive of $X$ is finite-dimensional in the sense of Kimura [13], then $X$ has a Chow-Künneth decomposition. Moreover, in characteristic 0, Kahn, Murre and Pedrini [11, Theorem 14.7.3] show that $X$ also has a Lefschetz decomposition. Since the class of varieties having finite-dimensional motives includes abelian varieties and is closed under quotients, the above constructions of Chow-Künneth and Lefschetz decompositions proceed by lifting them from a Weil cohomology theory to Chow groups in an inductive manner. However, these methods, although wider in scope, do not yield explicit simple formulae for the decompositions as in [1] and the present paper, which may be important in applications, as in [1, Theorem 1] and [11, Remark 14.6.13]. Moreover, our work does not place any restriction on the characteristic of the ground field.

It is easy to see that the projectors appearing in a Chow-Künneth or Lefschetz decomposition are not in general uniquely determined. However, for varieties whose motives are finite-dimensional, as in the case considered in this paper, the results of [11, Theorem 14.6.9, Theorem 14.7.3] imply that the motives defined by the projectors are uniquely determined up to isomorphism.

Acknowledgments. We thank the referee for his/her helpful comments that have improved the exposition.

\section{Terminology and Statements of Results}

First we recall the main result of [1], which we will need in our proof. As in [1], we work throughout in the category of rational Chow motives of pseudo-smooth projective varieties.

Theorem 2.1. [1, Theorem 1.1] Let $A$ be an abelian variety of dimension d over a field $k$ and $G$ a finite group acting on $A$. Let $f: A \longrightarrow A / G$ be the quotient map. Suppose $\left[\Delta_{A}\right]=\sum_{i=0}^{2 d} \pi_{i}$ is the Beauville-Deninger-Murre Chow-Künneth decomposition for $A$ and let $\eta_{i}=\frac{1}{|G|^{3}}(f \times f)_{*} \sum_{g, h \varepsilon G}(g, h)^{*} \pi_{i}$.

The

$$
\left[\Delta_{A / G}\right]=\sum_{i=0}^{2 d} \eta_{i}
$$

is a Chow-Künneth decomposition for $A / G$. This decomposition satisfies Poincaré duality: that is, for any $i$, $\eta_{2 d-i}={ }^{t} \eta_{i}$.

In addition, $\eta_{i}$ acts as zero on $C H_{\mathbb{Q}}^{j}(A / G)$ for $i<j$ and also for $i>j+d$ in general. In case $d \leq 4$, we may also conclude that $\eta_{i}$ acts trivially on $C H_{\mathbb{Q}}^{j}(A / G)$ for $i<j$ and also for $i>2 j$.

Next let $k$ be a field and $A$ an abelian variety of dimension $d$ over $k$. Let $G$ be a finite group acting on $A$, and let $f: A \rightarrow A / G$ denote the quotient map. We write $\Delta=\Delta_{A}: A \rightarrow A \times{ }_{k} A$ for the diagonal map on $A$.

Following Beauville [4], we set $C H_{s}^{i}(A, \mathbb{Q})=\left\{x \in C H^{i}(A, \mathbb{Q}): \mathbf{n}^{*} x=n^{2 i-s} x\right.$ for all $\left.n \neq 0, \pm 1\right\}$ and recall that 


$$
C H^{i}(A, \mathbb{Q})=\bigoplus_{s=i-d}^{i} C H_{s}^{i}(A, \mathbb{Q})
$$

Let $\mathcal{L}$ be an ample line bundle on $A$, and set $b=c_{1}(\mathcal{L})$. As in [14], Assumption 2.1, we may assume without loss of generality that

$$
b \varepsilon C H_{0}^{1}(A, \mathbb{Q})
$$

To see this, one may argue as follows: if $\sigma: A \rightarrow A$ is the involution $a \mapsto-a$, then $\mathcal{L}^{\prime}=\mathcal{L} \otimes \sigma^{*} \mathcal{L}$ is another ample line bundle on $A$, and $c_{1}\left(\mathcal{L}^{\prime}\right)=b+\sigma^{*} b$. Writing $b=b_{0}+b_{1}$ where $b_{0} \varepsilon C H_{0}^{1}(A, \mathbb{Q})$ and $b_{1} \varepsilon C H_{1}^{1}(A, \mathbb{Q})$, we have, for any $n \neq 0, \pm 1, \mathbf{n}^{*}\left(b_{1}+\sigma^{*} b_{1}\right)=\mathbf{n}^{*}\left(b_{1}\right)+(-\mathbf{n})^{*} b_{1}=n b_{1}-n b_{1}=0$, so that $b_{1}+\sigma^{*} b_{1}=0$. On the other hand, $\mathbf{n}^{*}\left(\sigma^{*} b_{0}\right)=(-\mathbf{n})^{*} b_{0}=(-n)^{2} b_{0}=n^{2} b_{0}$, so that $\sigma^{*} b_{0}=b_{0}$ and so $b+\sigma^{*} b=2 b_{0} \in C H_{0}^{1}(A, \mathbb{Q})$.

Define

$$
\begin{aligned}
\mathcal{L}_{G} & =\bigotimes_{g \varepsilon G} g^{*}(\mathcal{L}) \\
b_{G} & =c_{1}\left(\mathcal{L}_{G}\right)=\sum_{g \varepsilon G} c_{1}\left(g^{*} \mathcal{L}\right)=\sum_{g \varepsilon G} g^{*} c_{1}(\mathcal{L})=\sum_{g \varepsilon G} g^{*} b \\
L & =\Delta_{*}(b), \\
L_{G} & =\Delta_{*}\left(b_{G}\right)=\sum_{g \varepsilon G}(g \times g)^{*} L \\
L_{G G} & =\sum_{g, h \varepsilon G}(g \times h)^{*} L \\
\tilde{L} & =\frac{1}{|G|^{3}}(f \times f)_{*} L_{G G}
\end{aligned}
$$

Next let $F_{A}\left(F_{\hat{A}}\right)$ denote the Fourier transform on $A$ (respectively, $\hat{A}$ ). We then define

$$
\begin{aligned}
c_{G} & =b_{G}^{d-1} /(d-1) !, \\
\Lambda_{G} & =(-1)^{d}\left[{ }^{t} \Gamma_{\sigma}\right] \circ F_{\hat{A}} \circ \Delta_{*}\left(F_{A} \circ\left(c_{G}\right)\right) \circ F_{A}, \\
\Lambda_{G G} & =\sum_{g, h \varepsilon G}(g \times h)^{*}\left(\Lambda_{G}\right) \\
\tilde{\Lambda} & =\frac{1}{|G|^{3}}(f \times f)_{*} \Lambda_{G G}
\end{aligned}
$$

In the next section we prove the following theorem, although our primary interest is in the corollary that follows it.

Theorem 2.2. The data $\left(C H^{*}(A / G \times A / G ; \mathbb{Q}), \circ, \tilde{L}, \tilde{\Lambda},\left(\eta_{i}\right)_{i=0}^{2 d}\right)$ (where o denotes the composition of correspondences) defines a Lefschetz algebra in the sense of [14, section 4]. In particular, this implies that there exist projectors $\left\{q_{i, k} \mid i, k\right\}$ refining the projectors $\left\{\eta_{i} \mid i\right\}$ on $A / G$ such the following relations hold on the sub-algebra of $\left(C H^{*}(A / G \times A / G ; \mathbb{Q}), \circ\right)$ generated by these correspondences:

(a) $\tilde{L} \circ \eta_{i}=\eta_{i+2} \circ \tilde{L}$.

(b) $\tilde{\Lambda} \circ \eta_{i}=\eta_{i-2} \circ \tilde{\Lambda}$.

(c) $[\tilde{\Lambda}, \tilde{L}]=\tilde{\Lambda} \circ \tilde{L}-\tilde{L} \circ \tilde{\Lambda}=\sum_{i=0}^{2 d}(d-i) \eta_{i}$. 
These imply:

(i) $\sum_{k} q_{i, k}=\eta_{i}$ for each $i$.

(ii) $q_{i, k} \circ \eta_{j}=\eta_{j} \circ q_{i, k}=q_{i, k}$ if $i=j$ and 0 otherwise.

(iii) $q_{i, k}=0$ for $(i, k)$ not in $I=\{(i, k) \varepsilon \mathbb{Z} \times \mathbb{Z} \mid \max (0, i-d) \leq k \leq[i / 2]\}$.

(iv) $q_{i, k} \circ q_{j, l}=q_{i, k}$ if $i=j$ and $k=l$ and 0 otherwise.

(v) $q_{i, k} \circ \tilde{L}=\tilde{L} \circ q_{i-2, k-1}$.

(vi) $\tilde{\Lambda} \circ q_{i, k}=q_{i-2, k-1} \circ \tilde{\Lambda}$.

(vii) $\tilde{L} \circ \tilde{\Lambda} \circ q_{i, k}=k(g-i+k+1) q_{i, k}$.

(viii) $\tilde{\Lambda} \circ \tilde{L} \circ q_{i, k}=(k+1)(g-i+k) q_{i, k}$.

Corollary 2.3. Let $A$ be an abelian variety of dimension $d$ over a field $k$ and $G$ a finite group acting on $A$. Let $A / G$ be the quotient variety and let $h^{i}(A / G)=\eta_{i}$ denote the Chow-Künneth components constructed as in the theorem above. Define $L^{k} P^{i}(A / G)=\left(A / G, q_{i+2 k, k}\right)$. Then the following hold:

(i) For $0 \leq i \leq 2 d$, the Chow motive $h^{i}(A / G)=\left(A / G, \eta_{i}\right)$ has a Lefschetz decomposition

$$
h^{i}(A / G) \cong \bigoplus_{k=\max \{0, g-i\}}^{[i / 2]} L^{k} P^{i}(A / G)
$$

(ii) (Hard Lefschetz) The iterated composition of correspondences $\tilde{L}^{i}: h^{d-i}(A / G) \rightarrow h^{d+i}(A / G)$ is an isomorphism of Chow motives for all $0 \leq i \leq d$.

(iii) If $k$ is a finite field and $0 \leq j \leq d$, the map $g: C H^{j}(A / G, \mathbb{Q}) \rightarrow C H^{d-j}(A / G, \mathbb{Q})$ defined by $g(\alpha)=\tilde{L}^{d-2 j} \circ \alpha$ is an isomorphism.

We remark that assertions (i) and (ii) above follow immediately from Theorem 2.2 by Sections 4 and 5 of [14]; thus, we focus our attention on proving Theorem 2.2 and statement (iii) of Corollary 2.3.

Remark. In the literature, there are two distinct ways the Hard Lefschetz Theorem (for a Weil cohomology $H^{*}$ ) is stated: Let $X$ denote a smooth projective scheme of dimension $d$.

(i) The iterated cup product $[L]^{i}: H^{d-i}(X) \rightarrow H^{d+i}(X)$ with the first Chern class of an ample line bundle $L$ is an isomorphism for all $0 \leq i \leq d$ : see [14] and also [5].

(ii) The iterated cup product $\left[L^{\prime}\right]^{d-i}: H^{i}(X) \rightarrow H^{2 d-i}(X)$ with the class of a smooth generic hyperplane section $L^{\prime}$ is an isomorphism for all $0 \leq i \leq d$ : see [11, Theorem 14.7.3].

A generic smooth hyperplane section $H$ defines an effective divisor and the associated line bundle $\mathcal{L}$ will be ample. Moreover, its first Chern class $c_{1}(\mathcal{L})$ will be a class that identifies with the class of the hyperplane section $H$. Therefore (i) implies (ii).

In Corollary 2.3, the Hard Lefschetz isomorphism is given by the correspondence $\tilde{L}^{i}$, where

$$
\tilde{L}=\frac{1}{|G|^{3}}(f \times f)_{*} L_{G G}=\frac{1}{|G|^{3}}(f \times f)_{*} \sum_{g, h \in G}(g \times h)^{*} \Delta_{A *} L
$$

and $L=\Delta_{*} b$, where $b=c_{1}\left(\mathcal{L}_{G}\right)$ is also assumed to be symmetric, i.e. $\sigma^{*}(b)=b$, where $\sigma: A \rightarrow A$ is the involution $a \mapsto-a$. 
On the other hand, the version in [11] essentially states that the isomorphism is given by (a power of) the correspondence $\tilde{M}=\Delta_{A / G_{*}} i_{*} i^{*}[A / G]$, where $i: W \hookrightarrow A / G$ is a generic smooth hyperplane section with respect to some fixed embedding of $A / G$ in projective space. Viewing $W$ as a subvariety of $A / G, \tilde{M}=\Delta_{A / G_{*}}[W]$, and thus:

$$
\tilde{M}=\Delta_{A / G_{*}}[W]=\frac{1}{|G|^{2}}(f \times f)_{*}(f \times f)^{*} \Delta_{A / G_{*}}[W]=\frac{1}{|G|^{2}}(f \times f)_{*} \Delta_{A *} f^{*}[W]
$$

Although the projectors appearing in the decompositions provided by Corollary 2.3 and by [11, Theorem 14.7.3] might be different, the corresponding motives they define are unique up to isomorphism, as discussed in the last paragraph in the Introduction.

Corollary 2.3 applies to the following classes of examples considered in [1]:

\section{Examples 2.4.}

Symmetric products of abelian varieties. Let $X$ denote an abelian variety and $X^{n} / \Sigma_{n}$ the $n$-fold symmetric power of $X$. Observe that the action of $\Sigma_{n}$ is not in general free so that the quotient $X^{n} / \Sigma_{n}$ may not be smooth.

$(\mathcal{L})$ Example of Igusa. (See [10]) Let $X$ be an abelian variety over $k$, with $\operatorname{char}(k) \neq 2$. Let $t$ denote a point of order 2 on $X$. Define an action of $\mathbb{Z} / 2 \mathbb{Z}$ on $X \times X$ by : $(x, y) \mapsto(x+t,-y)$, and let $Y$ denote the quotient variety for this action. Now one sees easily that the action is free so that $Y$ is smooth; nevertheless, in positive characteristic, $Y$ need not be an abelian variety, as shown in [10]. If $X$ is an elliptic curve, the resulting surface $Y$ is a so-called bielliptic surface; see [4] VI, 19-20. In this case, the results of [17] also provide an explicit Chow-Künneth decomposition for $Y$.

(3) Kummer varieties. These are quotients of abelian varieties by the action of the group $\mathbb{Z} / 2 \mathbb{Z}$ where the group acts by identifying $a$ and $-a$.

Remark. In the case of desingularizations of the Kummer surfaces (as in (3) above), the results of [17] and $[11,14.6 .12]$ provide explicit projectors, with those in [11] refining the projectors in [17].

The techniques involved in our proof are extensions of those of [1]; the main advantage of this approach is that it yields explicit closed formulae for all the operators involved. In contrast, the construction of the refined projectors $\left\{q_{i, k} \mid i, k\right\}$ in [11] is an inductive one.

\section{Proofs}

3.1. Preliminaries. One of the key steps in proving the main theorem of [1] was to show that given any action $\alpha: G \times A \rightarrow A$ of a finite group $G$ on $A$, there exists an action $\beta: G \times A \rightarrow A$ of $G$ on $A$ with the following properties. First, the quotient of $A$ by the the first action of $G$ is isomorphic to the quotient of $A$ by the second action; second, for every $g$ in $G, \beta(g, 0)$ is a torsion point of $A$. This reduction will also be useful to us in the present article, so we assume henceforth that for every $g \varepsilon G, g(0)$ is a torsion point of $A$.

Now let $m_{g}$ be the order of $a_{g}=g(0)$. Next, let $m=\prod_{g \varepsilon G} m_{g}$, and

$$
E=\{n \varepsilon \mathbb{Z}: n \equiv 1(\bmod m), n \neq \pm 1\}
$$

Note that if $n \varepsilon E, m_{g}$ divides $n-1$ (for any $g$ ), so $n a_{g}=a_{g}$. 
For each $g \varepsilon G$, the map $g: A \rightarrow A$ defined by $a \mapsto g \cdot a$ factors uniquely as $g=\tau_{a_{g}} \circ g_{0}$, where $g_{0}: A \rightarrow A$ is a homomorphism, and $\tau_{a_{g}}: A \longrightarrow A$ is the translation $a \mapsto a+a_{g}$. Thus, if $n \varepsilon E$ and $a \in A,(\mathbf{n} \circ g)(a)=$ $n(g(a))=n\left(g_{0}(a)+a_{g}\right)=g_{0}(n a)+a_{g}=(g \circ \mathbf{n}) a ;$ that is,

$$
\mathbf{n} \circ g=g \circ \mathbf{n} .
$$

Recall our choice of ample line bundle $\mathcal{L}$ from Section 1. It follows from [8], Exercise II.7.5 that $\mathcal{L}_{G}=$ $\otimes_{g \varepsilon G} g^{*}(\mathcal{L})$ is also an ample line bundle. Moreover, because $b=c_{1}(\mathcal{L}) \varepsilon C H_{0}^{1}(A, \mathbb{Q}), \mathbf{n}^{*} b=n^{2} b$ for all $n \neq$ $0, \pm 1$. Now if $n \varepsilon E$ and $g \varepsilon G, \mathbf{n}^{*}\left(g^{*} b\right)=g^{*}\left(\mathbf{n}^{*} b\right)=n^{2} g^{*} b$. By the following result, this suffices to show that $g^{*} b \varepsilon C H_{0}^{1}(A, \mathbb{Q})$ and hence that $b_{G}=\sum_{g \varepsilon G} g^{*} b \varepsilon C H_{0}^{1}(A, \mathbb{Q})$ is a class associated to an ample line bundle and satisfies the hypothesis in 2.0.1.

Proposition 3.1. Let $E$ be as before, and let $\alpha \varepsilon C H^{1}(A, \mathbb{Q})$ be such that $\mathbf{n}^{*}(\alpha)=n^{2}(\alpha)$ for all neE. Then $\alpha \circ \pi_{2}=\alpha$ and $\alpha \circ \pi_{j}=0$ for all $j \neq 2$ and hence $\alpha \varepsilon C H_{0}^{1}(A, \mathbb{Q})$.

Proof. This is a standard computation:

$$
\begin{aligned}
\mathbf{n}^{*}(\alpha) & =\mathbf{n}^{*}\left(\alpha \circ\left[\Delta_{A}\right]\right) \\
& =\sum_{j=0}^{2 d} \mathbf{n}^{*}\left(\alpha \circ \pi_{j}\right) \\
& =\sum_{j=0}^{2 d} \mathbf{n}^{*}\left(p_{2 *}\left(p_{1}^{*}(\alpha) \bullet \pi_{j}\right)\right. \\
& =\sum_{j=0}^{2 d} p_{2 *}\left(p_{1}^{*}(\alpha) \bullet(1 \times \mathbf{n})^{*}\left(\pi_{j}\right)\right) \\
& =\sum_{j=0}^{2 d} p_{2 *}\left(p_{1}^{*}(\alpha) \bullet n^{j} \pi_{j}\right) \\
& =\sum_{j=0}^{2 d} n^{j}\left(\alpha \circ \pi_{j}\right)
\end{aligned}
$$

Therefore, we obtain:

$$
n^{2}\left(\alpha-\alpha \circ \pi_{2}\right)+\sum_{j \neq 2} n^{j}\left(\alpha \circ \pi_{j}\right)=0
$$

for all $n \varepsilon E$. Since $E$ is an infinite subset of the integers, the required conclusion follows.

3.2. Technical Lemmas. We now make some general computations which will be helpful in proving the main theorem. We will use the following lemma repeatedly in the sequel, often without explicit mention.

Lemma 3.2. Let $g, h \varepsilon G$ and $\alpha, \beta \varepsilon C H^{*}\left(A \times_{k} A, \mathbb{Q}\right)$. Then for any $k \varepsilon G$,

(i) $(g \times h)^{*} \alpha \circ \beta=(k \times h)^{*}\left(\alpha \circ\left(k^{-1} \times g^{-1}\right)^{*} \beta\right)$

(ii) $\alpha \circ(g \times h)^{*} \beta=(g \times k)^{*}\left(\left(h^{-1} \times k^{-1}\right)^{*} \alpha \circ \beta\right)$

(iii) $(g \times g)^{*}(\alpha \circ \beta)=(g \times g)^{*} \alpha \circ(g \times g)^{*} \beta$. 


\section{Proof.}

The third statement follows from the first by taking $h=k=g$ and replacing $\beta$ by $(g \times g)^{*} \beta$. We prove only the first statement, as the second is similar.

$$
\begin{aligned}
& (g \times h)^{*} \alpha \circ \beta=p_{13 *}\left(p_{12}^{*} \beta \bullet p_{23}^{*}(g \times h)^{*} \alpha\right)=p_{13 *}\left(p_{12}{ }^{*} \beta \bullet(k \times g \times h)^{*} p_{23}^{*} \alpha\right) \\
= & p_{13 *}(k \times g \times h)^{*}\left(\left(k^{-1} \times g^{-1} \times h^{-1}\right)^{*} p_{12} * \beta \bullet p_{23}^{*} \alpha\right)=p_{13 *}\left(k^{-1} \times g^{-1} \times h^{-1}\right)_{*}\left(p_{12}{ }^{*}\left(k^{-1} \times g^{-1}\right)^{*} \beta \bullet p_{23}{ }^{*} \alpha\right) \\
& =\left(k^{-1} \times h^{-1}\right)_{*} p_{13 *}\left(p_{12}{ }^{*}\left(k^{-1} \times g^{-1}\right)^{*} \beta \bullet p_{23}{ }^{*} \alpha\right)=(k \times h)^{*} p_{13 *}\left(p_{12}{ }^{*}\left(k^{-1} \times g^{-1}\right)^{*} \beta \bullet p_{23}{ }^{*} \alpha\right) \\
= & (k \times h)^{*}\left(\alpha \circ\left(k^{-1} \times g^{-1}\right)^{*} \beta\right)
\end{aligned}
$$

Following the notation of (2.0.2), we define, for $\alpha \in C H^{*}(A \times A, \mathbb{Q}), \alpha_{G}=\sum_{g \varepsilon G}(g \times g)^{*} \alpha$ and $\alpha_{G G}=\sum_{g, h \varepsilon G}(g \times h)^{*} \alpha$. Observe that $\alpha_{G G}=\sum_{g, h \varepsilon G}(g \times h)^{*} \alpha=\sum_{g \varepsilon G}(g \times 1)^{*}\left(\sum_{h \varepsilon G}(h \times h)^{*} \alpha\right)=\sum_{g \varepsilon G}(g \times 1)^{*} \alpha_{G}$ and similarly $\alpha_{G G}=\sum_{h \varepsilon G}(1 \times h)^{*} \alpha_{G}$.

Lemma 3.3. If $\alpha, \beta \varepsilon C H^{*}(A \times A, \mathbb{Q})$, then $\alpha_{G G} \circ \beta_{G G}=\left(\alpha_{G} \circ \beta_{G}\right)_{G G}$.

Proof. From the equations above,

$$
\begin{gathered}
\alpha_{G G} \circ \beta_{G G}=\sum_{h \varepsilon G}(1 \times h)^{*} \alpha_{G} \circ \sum_{g \varepsilon G}(g \times 1)^{*} \beta_{G} \\
=\sum_{g, h \varepsilon G} p_{13 *}\left(p_{12}{ }^{*}(g \times 1)^{*} \beta_{G} \bullet p_{23}{ }^{*}(1 \times h)^{*} \alpha_{G}\right) \\
=\sum_{g, h \varepsilon G} p_{13 *}(g \times 1 \times h)^{*}\left(p_{12}{ }^{*} \beta_{G} \bullet p_{23}{ }^{*} \alpha_{G}\right) \\
=\sum_{g, h \varepsilon G}(g \times h)^{*} p_{13 *}\left(p_{12}{ }^{*} \beta_{G} \bullet p_{23}{ }^{*} \alpha_{G}\right)=\sum_{g, h \varepsilon G}(g \times h)^{*}\left(\alpha_{G} \circ \beta_{G}\right)=\left(\alpha_{G} \circ \beta_{G}\right)_{G G}
\end{gathered}
$$

3.3. Proof of Theorem 2.2. By [1, Lemma 2.4], the elements $\rho_{i}=\sum_{g, h \varepsilon G}(g, h)^{*} \pi_{i}=\left(\pi_{i}\right)_{G G} \varepsilon C H^{d}(A \times$ $A, \mathbb{Q}))^{G \times G}, 0 \leq i \leq 2 d$ satisfy $\rho_{i} \circ \rho_{i}=|G|^{2} \rho_{i}$ and $\rho_{i} \circ \rho_{j}=0$ if $i \neq j$.

Since $\mathcal{L}_{G}$ is a symmetric ample line bundle (i.e. its first Chern class satisfies the condition in (2.0.1)), the main result of [14] implies directly that

$$
\left(C H^{*}(A \times A, \mathbb{Q}), \circ, L_{G}, \Lambda_{G},\left(\pi_{i}\right)_{i=0}^{2 d}\right) \text { is a Lefschetz algebra. }
$$

In particular, the following hold (for any $j$ ):

$$
\begin{gathered}
L_{G} \circ \pi_{j}=\pi_{j+2} \circ L_{G} \\
\Lambda_{G} \circ \pi_{j}=\pi_{j-2} \circ \Lambda_{G} \\
{\left[\Lambda_{G}, L_{G}\right]=\sum_{i=0}^{2 d}(d-i) \pi_{i}}
\end{gathered}
$$

In this setting, the following result may be viewed as an equivariant analogue of the statement (3.3.1).

Lemma 3.4. The following properties hold: 
(i) $C H^{*}(A \times A, \mathbb{Q})^{G \times G}$ is a graded $\mathbb{Q}$-algebra with unit element $\frac{1}{|G|^{2}}\left[\Delta_{A}\right]_{G G}$.

(ii) $\sum_{i=0}^{2 d} \rho_{i}=\left[\Delta_{A}\right]_{G G}$

(iii) $\rho_{i} \circ \rho_{j}= \begin{cases}|G|^{2} \rho_{i} & \text { if } i=j \\ 0 & \text { otherwise }\end{cases}$

(iv) $L_{G G} \circ \rho_{i}=\rho_{i+2} \circ L_{G G}$

(v) $\Lambda_{G G} \circ \rho_{i}=\rho_{i-2} \circ \Lambda_{G G}$

(vi) $\left[\Lambda_{G G}, L_{G G}\right]=|G|^{2} \sum_{i=0}^{2 d}(d-i) \rho_{i}$.

\section{Proof.}

Lemma 3.2 shows that $C H^{*}(A \times A, \mathbb{Q})^{G \times G}$ is a ring under cycle addition and composition of correspondences, with unit element $\frac{1}{|G|^{2}}\left[\Delta_{A}\right]_{G G}$. The second and third statements follow from the construction of the $\rho_{i}$. It remains to prove that last three statements.

By construction, $\rho_{i}=\left(\pi_{i}\right)_{G G}$. By Lemmas 3.2 and 3.3, we have

$$
L_{G G} \circ \rho_{i}=L_{G G} \circ\left(\pi_{i}\right)_{G G}=\left(L_{G} \circ\left(\pi_{i}\right)_{G}\right)_{G G}=\left(L_{G} \circ \sum_{g \varepsilon G}(g \times g)^{*} \pi_{i}\right)_{G G}=\sum_{g \varepsilon G}\left((g \times g)^{*}\left(L_{G} \circ \pi_{i}\right)\right)_{G G}
$$

On the other hand,

$$
\rho_{i+2} \circ L_{G G}=\left(\pi_{i+2}\right)_{G G} \circ L_{G G}=\left(\left(\pi_{i+2}\right)_{G} \circ L_{G}\right)_{G G}=\sum_{g \varepsilon G}\left[(g \times g)^{*} \pi_{i+2} \circ L_{G}\right]_{G G}=\sum_{g \varepsilon G}\left[(g \times g)^{*}\left(\pi_{i+2} \circ L_{G}\right)\right]_{G G}
$$

By (3.3.2), the two right-hand-sides are equal, thereby proving the fourth statement. The proof of the fifth statement is virtually identical.

It remains to prove the commutator relation. Again, using Lemmas 3.2 and 3.3 together with (3.3.4),

$\left[\Lambda_{G G}, L_{G G}\right]=\Lambda_{G G} \circ L_{G G}-L_{G G} \circ \Lambda_{G G}=\left(\Lambda_{G} \circ L_{G}\right)_{G G}-\left(L_{G} \circ \Lambda_{G}\right)_{G G}=\left[\Lambda_{G}, L_{G}\right]_{G G}=\sum_{i=0}^{2 d}(d-i)\left(\pi_{i}\right)_{G G}=\sum_{i=0}^{2 d}(d-i) \rho_{i}$

This concludes the proof of Lemma 3.4.

We now continue with the proof of Theorem 2.2. As in [1, Proposition 3.4] the projections $A \times A \times A \rightarrow A \times A$ and $A \times A \rightarrow A$ will be denoted $p$ with the appropriate superscripts and subscripts to indicate which factors are the source and the target. (For example, $p_{23}^{123}$ will denote the projection $A \times A \times A \rightarrow A \times A$ projecting to the second and third factors.) The corresponding projections for $A / G$ will be denoted $q$ with the corresponding indices. For convenience of notation set $r=(f \times f \times f): A \times_{k} A \times_{k} A \longrightarrow A / G \times_{k} A / G \times_{k} A / G$.

Lemma 3.5. Let $\alpha, \beta \varepsilon C H^{*}(A \times A, \mathbb{Q})^{G \times G}$. Then $(f \times f)_{*}(\alpha) \circ(f \times f)_{*}(\beta)=|G|(f \times f)_{*}(\alpha \circ \beta)$. In particular, $\left((f \times f)_{*}(\alpha)\right)^{i}=|G|^{i-1}(f \times f)_{*}\left(\alpha^{i}\right)$, where the exponent $i$ denotes the $i$-fold iterated composition of correspondences.

Proof. Now,

$$
(f \times f)_{*}(\alpha) \circ(f \times f)_{*}(\beta)=q_{13}^{123}\left(q_{12}^{123^{*}}(f \times f)_{*}(\alpha) \bullet q_{23}^{123^{*}}(f \times f)_{*}(\beta)\right)
$$

Since the degree of $r$ is $|G|^{3}, r_{*} r^{*}$ corresponds to multiplication by $|G|^{3}$, and therefore, the last expression equals: 


$$
\frac{1}{|G|^{3}} q_{13}^{123}\left(r_{*} r^{*} q_{12}^{123^{*}}(f \times f)_{*}(\alpha) \bullet q_{23}^{123^{*}}(f \times f)_{*}(\beta)\right)
$$

Because $q_{12}^{123} \circ r=(f \times f) \circ p_{12}^{123}$, the above simplifies to:

$$
\frac{1}{|G|^{3}} q_{13}^{123}{ }_{*}\left(r_{*} p_{12}^{123^{*}}(f \times f)^{*}(f \times f)_{*}(\alpha) \bullet q_{23}^{123^{*}}(f \times f)_{*}(\beta)\right)
$$

Since $\alpha$ is $G \times G$-invariant, $(f \times f)^{*}(f \times f)_{*}$ is multiplication by $|G|^{2}$, so the expression equals:

$$
\frac{1}{|G|^{3}} q_{13}^{123}\left(r_{*} p_{12}^{123^{*}}|G|^{2} \alpha \bullet q_{23}^{123^{*}}(f \times f) *(\beta)\right)
$$

Finally, applying the projection formula, the formula $q_{i j}^{123} \circ r=(f \times f) \circ p_{i j}^{123}$ and $(G \times G)$-invariance of both $\alpha$ and $\beta$, one may identify the last expression with:

$$
\begin{aligned}
\frac{1}{|G|} q_{13}^{123}{ }_{*} r_{*}\left(p_{12}^{123^{*}}(\alpha) \bullet r^{*} q_{23}^{123^{*}}(f \times f)_{*}(\beta)\right) & =\frac{1}{|G|}(f \times f)_{*} p_{13}^{123}\left(p_{12}^{123^{*}}(\alpha) \bullet p_{23}^{123^{*}}(f \times f)^{*}(f \times f)_{*}(\beta)\right) \\
& =|G|(f \times f)_{*} p_{13}^{123}\left(p_{12}^{123^{*}}(\alpha) \cdot p_{23}^{123 *}(\beta)\right) \\
& =|G|(f \times f)_{*}(\alpha \circ \beta)
\end{aligned}
$$

This proves the first statement of the lemma and the second follows readily.

Now define $\tilde{L}=\frac{1}{|G|^{3}}(f \times f)_{*}\left(L_{G G}\right), \tilde{\Lambda}=\frac{1}{|G|^{3}}(f \times f)_{*}\left(\Lambda_{G G}\right)$, and $\eta_{i}=\frac{1}{|G|^{3}}(f \times f)_{*}\left(\rho_{i}\right)$. Lemmas 3.5 and 3.4 together imply that $\left(C H^{*}(A / G), \tilde{L}, \circ, \tilde{\Lambda},\left(\eta_{i}\right)_{i=0}^{2 d}\right)$ forms a Lefschetz algebra, thus concluding the proof of Theorem 2.2.

The first two assertions of Corollary 2.3 follow immediately from the formalism of Sections 4 and 5 of [14]. Finally, we suppose that $k$ is a finite field and prove statement (iii) of Corollary 2.3. Since $L_{G G}$ is $G \times G$ invariant, Lemma 3.2 shows that the map $\phi: C H^{p}(A ; \mathbb{Q}) \rightarrow C H^{d-p}(A, \mathbb{Q})$ defined by $\alpha \mapsto L_{G G}^{d-2 p} \circ \alpha$ leaves the $G$-invariant part-stable: i.e. $\phi$ restricts to a map

$$
\phi^{G}: C H^{p}(A, \mathbb{Q})^{G} \rightarrow C H^{d-p}(A, \mathbb{Q})^{G}
$$

Observe that the projection $\pi: C H^{*}(A, \mathbb{Q}) \rightarrow C H^{*}(A, \mathbb{Q})^{G}$ sends $\alpha \mapsto \sum_{g \varepsilon G} g^{*}(\alpha)$. Thus one may readily verify the commutativity of the two squares:

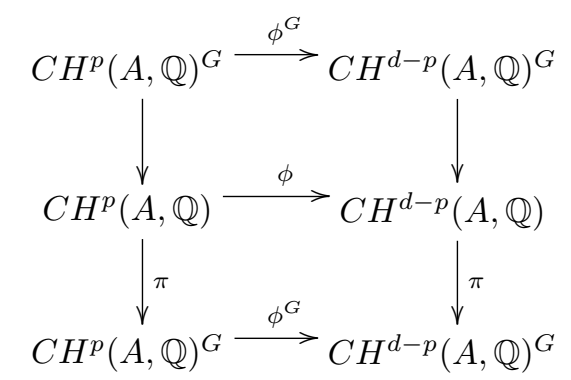

The middle row is an isomorphism by the Hard Lefschetz Theorem for the Chow groups of abelian varieties as proved in [14, Theorem 5.2]. Hence the top row is injective and the bottom row is surjective, thereby proving statement (iii) in Corollary 2.3. 


\section{REFERENCES}

[1] R. Akhtar and R. Joshua, Kunneth decomposition for quotient varieties, Indag. Math. 17 (2006), no. 3, 319-344.

[2] Y. André, B. Kahn and P. O'Sullivan, Nilpotence, radicaux et structures monoïdales, Rend. Sem. Mat. Univ. Padova 108 (2002), 107-291.

[3] D. Arapura, Motivation for Hodge cycles, Preprint (to appear in Advances in Math), (2006)

[4] A. Beauville, Quelques remarques sur la transformation de Fourier dans l'anneau de Chow d'une variété abélienne. Lect. Notes. in Math. 1016 (1983), pp. 238-260.

[5] P. Deligne, La conjecture de Weil. II, Inst. Hautes tudes Sci. Publ. Math. No. 52 (1980), pp. 137-252.

[6] C. Deninger and J. Murre, Motivic decomposition of Abelian schemes and the Fourier transform. J. reine und angew. Math. 422 (1991), pp. 201-219.

[7] V. Guletskii and C. Pedrini, Finite dimensional motives and the conjectures of Beilinson and Murre, K-Theory, 30, (2003), no. 3, 243-263.

[8] R. Hartshorne, Algebraic Geometry, Springer-Verlag, 1977.

[9] P. J. Hilton and U. Stammbach, Homological Algebra, Springer, GTM, (1972)

[10] J-I. Igusa, On some problems in abstract algebraic geometry. Proc. Acad. Nat. Sci. USA 41 (1955), pp. 964-967.

[11] B. Kahn, J. Murre and C. Pedrini, On the transcendental part of the motive of a surface, preprint, (2005)

[12] S. Kleiman, "The Standard Conjectures", in Motives, Proc. Symp. Pure Math. 55 part 1, AMS (1994), pp. 3-20.

[13] S-I. Kimura, Chow groups can be finite dimensional, in some sense, Math. Annalen, 331, 173-201 (2005)

[14] K. Künnemann, A Lefschetz decomposition for Chow motives of Abelian schemes, Invent. Math., 113, 85-102, (1993)

[15] Y. Manin, Correspondences, motives, and monoidal transformations, Math. USSR-Sb. 6 (1968), pp. 439-470.

[16] D. Mumford, Abelian varieties. Tata Institute of Fundamental Research Studies in Mathematics, No.5. Oxford University Press (1970).

[17] J. Murre, On the motive of an algebraic surface, J. Reine Angew. Math. 409 (1990). pp. 190-204.

[18] J. Murre, On a conjectural filtration on the Chow groups of an algebraic variety: I and II. Indag. Math. (N.S.) 4 (1993), no. 2, pp. 177-201.

[19] A. J. Scholl, "Classical motives" in Motives, Proc. Symp. Pure Math. 55 part 1, AMS (1994), pp. 189-205.

[20] A. M. Shermenev, The motive of an abelian variety. Funct. Anal. 8 (1974), pp. 55-61.

Department of Mathematics, Miami University, Oxford, Ohio, 45056, USA.

E-mail address: reza@calico.mth.muohio.edu

Department of Mathematics, Ohio State University, Columbus, Ohio, 43210, USA.

E-mail address: joshua@math.ohio-state.edu 